\title{
Precision-guided genetic ordnance: new developments in receptor targeting of recombinant AAV
}

TR Flotte

Gene Therapy (2003) 10, 2051. doi:10.1038/sj.Gt.3302161

The appeal of receptor-mediated gene transfer has always stemmed from its precisely defined nature. In a paradigm similar to structurally oriented drug design, ligands are usually chosen based on their affinity for well-characterized receptors that are displayed on the target cell. The drawback of this approach in its original incarnation as a proteinplasmid conjugate is the relatively low efficiency of transduction and the lack of any intrinsic mechanism for long-term persistence. The recent emergence of techniques allowing for the incorporation of peptides into capsid proteins of viral vectors like recombinant adeno-associated virus (rAAV) opened the door to the possibility of exploiting receptorspecific targeting, while retaining the efficiency and stability conferred by the viral vector. Several proofs of this concept have been published in recent years, ${ }^{1-3}$ although the success of such peptide-displaying vectors has been somewhat hit-and-miss. Particularly at issue is the fact that the number of known ligands small enough for such a strategy is quite limited for some cell types.

A recent report by Müller et $a l^{4}$ provides a paradigm for overcoming that limitation, paradoxically by using an empiric random screening approach to select ligands of high specificity. This group has developed a method for incorporating random small (7-mer) peptides within the heparan-binding loop on the exterior surface of the AAV capsid. They were thus able to create a diverse library of receptor-targeted rAAV constructs, and to screen these constructs for their ability to infect coronary endothelial cells - a target cell for gene transfer that is not very permissive for wild-type AAV2 infection. By passaging on this cell type, the group was able to select for capsid mutants that contained peptide ligands of interest. They then verified the utility of these targeted vectors by packaging a reporter gene into the capsids, confirming their efficacy on this very important target cell type.

The immediate implication of this report is the availability of rAAV vectors to target coronary artery endothelial cells. While natural rAAV serotypes have been found to be quite efficient for gene transfer into cells such as myofibers, neurons, photoreceptors and hepatocytes, endothelial cells have proven to be a particularly resistant target. The concept of using targeting to overcome this obstacle was earlier described by Nicklin et $a l,{ }^{5}$ who demonstrated that the SIGYPLP heptapeptide increased gene transfer efficiency dramatically in some endothelial cells. The Müller study greatly expands the number of such ligands available, which is important because endothelial cells derived from different segments of the circulation (venules, capillaries, arterioles), and from within different organs, appear to demonstrate unique receptor phenotypes.

Furthermore, the peptide display libraries offer an approach that could be more generally applicable to other relatively resistant cell types, such as certain hematopoietic cells, including hematopoietic stem cells. Thus, the potential for an efficiently targeted gene vector for the target cell of one's choice appears to be greatly improved.

It is important to note that the role of targeting could be of importance even for cell types that are permissive for transduction by existing rAAV serotypes. The consideration here is that once the move to in vivo gene transfer is made, it becomes desirable for gene transfer not only to be efficient, but also to be very selective. One example of this is the recent concern raised regarding the appearance of rAAV2 genomes in the semen of male hemophiliacs receiving hepatic delivery of a rAAV2-factor IX vector. Even if a high efficiency of gene transfer could be achieved, the prohibition on germline gene transfer would make it very problematic to proceed, should it ever be demonstrated that rAAV were present within spermatocytes themselves. Thus, a more selectively targeted rAAV could be of immediate clinical relevance in this situation.

Finally, one can envision applications in which selective targeting is not only desirable because of theoretical concerns such as the germline issue. For example, a vector selectively targeted to a tumor cell antigen might deliver a prodrugactivating enzyme or some other suicide gene, selectively, to the offending tumor cells. Such selectivity is the essence of the mythical 'silver bullet' that kills only undesirable targets, but is completely nontoxic to innocent bystanders. While the current state does not yet reach this level of selectivity and efficiency, the field is marking a clear path toward that goal.

Terence $R$ Flotte is the Nemours Eminent Scholar and Chair of Pediatrics at the University of Florida College of Medicine, USA. E-mail: flotttr@peds.ufl.edu

1 Bartlett JS, Kleinschmidt J, Boucher RC, Samulski RJ. Targeted adeno-associated virus vector transduction of nonpermissive cells mediated by a bispecific F(ab'gamma) antibody. Nat Biotechnol 1999; 17: 181-186.

$2 \mathrm{Wu} \mathrm{P}$ et al. Mutational analysis of the adeno-associated virus type 2 (AAV2) capsid gene, construction of AAV2 vectors with altered tropism. I Virol 2000; 74: 8635-8647.

3 Yang $Q$ et al. Development of novel cell surface CD34-targeted recombinant adenoassociated virus vectors for gene therapy. Hum Gene Ther 1998; 9: 1929 1937.

4 Müller OJ et al. Random peptide libraries displayed on adeno-associated virus to select for targeted gene therapy vectors. Nat Biotechnol 2003; 9: 1040-1046.

5 Nicklin SA et al. Efficient and selective AAV2-mediated gene transfer directed to human vascular endothelial cells. $\mathrm{Mol}$ Ther 2001; 4: 174-181. 